\title{
RMA2 Background Correction Method
}

National Cancer Institute

\section{Source}

National Cancer Institute. RMA2 Background Correction Method. NCI Thesaurus. Code C64236.

Perform a modified version of RMA background correction. Defined in the documents available at http://bioconductor.org/packages/1.9/bioc/html/affy.html. 